\title{
APPLICATION OF AFFINE NARMA MODEL TO DESIGN OF ADAPTIVE POWER SYSTEM STABILIZER
}

\author{
Jingsen ZHOU ${ }^{1}$, Deping KE ${ }^{1}$, Chi Yong CHUNG $^{2}$, Yuanzhang SUN ${ }^{1}$ \\ ${ }^{1}$ School of Electrical Engineering, Wuhan University, Wuhan, Hubei Province, China, 430072; \\ zhoujingsen_whu@163.com \\ ${ }^{2}$ Department of Electrical and Computer Engineering, University of Saskatchewan, Saskatoon, \\ Saskatchewan, Canada,
}

\begin{abstract}
An affine nonlinear autoregressive moving average (NARMA) model is derived from the neural network $(\mathrm{NN})$ based general NARMA model in this paper, by using Taylor series expansion. The predictive error of this affine NARMA model will be quite acceptable, at least for the control purpose, if the amplitude of control input is properly limited. Therefore, an adaptive control scheme based on this model is proposed and applied to the design of adaptive power system stabilizer (APSS) since the amplitude of PSS output is usually well limited. The feature of this control scheme is that the control input can be online analytically obtained. Thus, comparing to the traditional NN based APSS (TAPSS), the affine NARMA model based APSS (AAPSS) does not need the training of a $\mathrm{NN}$ as neuro-controller, which may be a troublesome and time consuming step during the design. Moreover, the AAPSS can generally perform better than the TAPSS. Simulation studies on a single machine infinite bus system and a multi-machine system show that the AAPSSs can consistently well perform to damp electromechanical oscillations in the systems over a wide range of operating conditions.
\end{abstract}

Keywords: adaptive control; affine; NARMA model; neural network; power system stabilizer (PSS)

\section{INTRODUCTION}

Power system stabilizers (PSSs) are accepted as the most effective devices to damp the low frequency oscillations in power systems. The conventional PSS (CPSS) is designed based upon a linearized model around a specific operating condition [7]. The performance of CPSSs, therefore, may significantly deteriorate due to the nonlinearities of system and the variation of operating condition $[1,4]$.

So far many neural network $(\mathrm{NN})$ based techniques have been proposed for the adaptive PSS (APSS) design to handle the nonlinear and non-autonomous characteristics of power systems. One type of NN based APSS is a controller that synthesizes the function of the PSSs which are designed in different operating conditions and with different disturbances $[1,10,16,20]$. Depending on a set of training data generated by the CPSSs $[1,10$, 16], or other types PSSs [20], a NN is first trained off-line and then acts online as an APSS. In order to ensure the universal adaption capability of the APSS, a wide range of operating conditions as well as various disturbances have to be considered in the design.

Another type of NN based APSS adopts the structure of indirect adaptive control [2, 3, 6, 14, 17, 19], where an identifier is used to online predict the output of the generator and the control law is established based upon this identifier. The $\mathrm{NN}$ identifiers can track the generator dynamic accurately and adaptively irrespective of operating condition by online updating their weights depending on the predictive error $[2,3,6,14,17]$. However, these identifiers are general nonlinear autoregressive moving average (NARMA) models. Thus, an additional NN is required to act as the neuro-controller, learning the optimal control law off-line [2]. Nevertheless, this learning process is generally quite troublesome and time-consuming; it also suffers the risk of converging to local minimum which may make the control performance unacceptable. Neuro-fuzzy controllers have been proposed to incorporate control experiences as fuzzy rules [3, 12, 19]. Therefore, the learning process of these controllers may be more efficient than that of the neuro-controllers with random initial weights.

It is shown in [9] that using an approximate model for the control design may have a better control effect than that obtained from a more accurate model. This is because the control method implemented on the approximate model can more adequately consider the structural characteristics of the model. In [13], the pole shift (PS) control algorithm is applied based on a linear autoregressive moving average (ARMA) model which approximates the dynamic of the nonlinear plant. However, since the generation of control input has to solve a nonlinear optimization problem, this control algorithm may not be fast enough for 
online implementation. Compared with the linear ARMA model, the NN based NARMA model can describe the dynamics of the nonlinear plant more accurately. However, because of the nonlinear relationship between the plant output and input, the solution of optimal control performance cannot be online calculated analytically, which will increase the computation burden in learning process of controller. Meanwhile, the affine transformation about NN based NARMA model, which makes the control design more tractable and efficient, has not been reported in the recent researches.

In this paper, an affine NARMA model is proposed for the control design. This model is obtained via implementing Taylor series expansion on the NN based general NARMA model. As long as the amplitude of the control input is properly limited, the affine NARMA can provide satisfactory prediction of the plant output, at least for the control purpose. Based on this model, an adaptive control scheme where the control input can be analytically computed online is proposed and applied to the design of APSS because the amplitude of the supplementary exciting control input is generally well confined. This affine NARMA model based APSS (AAPSS) can generally perform better than the traditional NN based APSS (TAPSS) [2, 3, 6, 14, 17]. Moreover, the AAPSS does not need to train a NN as neurocontroller which is required by the TAPSS.

The paper is structured as follows: Section 2 introduces the NN based general NARMA model and the proposed affine NARMA model. The proposed adaptive control scheme is applied to the design of APSS in Section 3. Section 4 gives comparisons of the AAPSS and the TAPSS. Simulation results are reported in Section 5 . Conclusions are presented in Section 6.

\section{NN BASED GENERAL NARMA MODEL AND PROPOSED AFFINE NARMA MODEL}

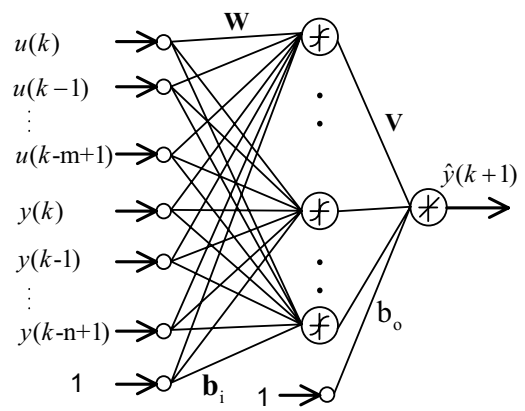

Fig. 1. Three-layer feedforward network

In $\mathrm{NN}$ based nonlinear control, a three-layer feedforward network (Fig. 1) is generally employed to identify the dynamic of a single-input-singleoutput plant [15]. This identifier (predictor) can be mathematically expressed by a general NARMA model as follows:

$$
\hat{y}(k+1)=\mathbf{V} \boldsymbol{\Phi}\left(\mathbf{W}\left[\mathbf{U}(k)^{\mathrm{T}} \mathbf{Y}(k)^{\mathrm{T}}\right]^{\mathrm{T}}+\mathbf{b}_{\mathrm{i}}\right)+\mathrm{b}_{\mathrm{o}}
$$

where $\hat{y}(k+1)$ is the predicted plant output at time step $k+1 ; \mathbf{U}(k)$ and $\mathbf{Y}(k)$ are input vectors to the network with the following definitions:

$$
\begin{gathered}
\mathbf{U}(k)=[u(k), u(k-1), \ldots, u(k-\mathrm{m}+1)]^{\mathrm{T}} \\
\mathbf{Y}(k)=[y(k), y(k-1), \ldots, y(k-\mathrm{n}+1)]^{\mathrm{T}}
\end{gathered}
$$

where $y(k)$ and $u(k)$ are plant output and control input, respectively, at time step $k ; \mathbf{W}$ and $\mathbf{V}$ are input weights and layer weights, respectively, of the network; $\mathbf{b}_{\mathrm{i}}$ and $\mathrm{b}_{\mathrm{o}}$ are biases in hidden layer and in output layer, respectively; $\boldsymbol{\Phi}$ is the function vector defined as follows:

$$
\boldsymbol{\Phi}=\left[\phi\left(x_{1}\right), \phi\left(x_{2}\right), \ldots, \phi\left(x_{\mathrm{p}}\right)\right]^{\mathrm{T}}
$$

where $\mathrm{p}$ denotes the number of neurons in the hidden layer; $\phi$ is the activation function and tansig function is used in this paper as follows:

$$
\phi\left(x_{\mathrm{p}}\right)=2 /\left(e^{-2 x_{\mathrm{p}}}+1\right)-1
$$

It has been demonstrated that a well designed and trained NN identifier can accurately predict the plant output [11]. In other words, the dynamics of the nonlinear plant in a neighborhood of the equilibrium can be exactly described by the $\mathrm{NN}$ based general NARMA model, such as (1). Nevertheless, the control effect is not only related with the accuracy of the model, but also depends on the control method implemented on the model [5]. For example, due to the special nonlinear structure, the gradient descent method is almost the exclusive control method for (1), but the control effect may be unacceptable because of the intrinsic limitations of the method (and this will be addressed in detail in the later section). Comparatively, a well established control technique can be implemented on a model with specific structure which may only approximately describe the dynamic of the plant. Consequently, a satisfactory control effect can be achieved because the controller synthesis makes full use of the structural characteristics of the model, such as the PS control algorithm implemented on the linear ARMA model [13].

For above considerations, an affine NARMA model is proposed in this paper and by taking advantage of the structural characteristic of this approximate model, an online adaptive control scheme is also developed. Expand the right hand side of (1) as Taylor series around $u(k)=0$ as follows:

$$
\hat{y}(k+1)=g_{0}(k)+g_{1}(k) \cdot u(k)+\text { H.O.T. }
$$

where H.O.T. denotes high order terms; $\mathrm{g}_{0}(k)$ and $\mathrm{g}_{1}(k)$ are calculated as follows:

$$
\mathbf{U}_{0}(k)=[0, u(k-1), \ldots, u(k-\mathrm{m}+1)]^{\mathrm{T}}
$$




$$
\begin{gathered}
\mathbf{X}=\mathbf{W}\left[\mathbf{U}_{0}(k)^{\mathrm{T}} \mathbf{Y}(k)^{\mathrm{T}}\right]^{\mathrm{T}}+\mathbf{b}_{\mathrm{i}} \\
\mathrm{g}_{0}(k)=\mathbf{V} \boldsymbol{\Phi}(\mathbf{X})+\mathrm{b}_{\mathrm{o}} \\
\mathrm{g}_{1}(k)=4 \mathbf{w}_{1} \cdot \mathbf{V}\left(e^{-2 \mathbf{x}} /\left(1+e^{-2 \mathbf{X}}\right)^{.2}\right)
\end{gathered}
$$

where $\mathbf{w}_{1}$ represents the first column of $\mathbf{W} ; \cdot$, ./ and 2 denotes dot production, dot division and dot square, respectively. It is proved that H.O.T. is bounded by [9]:

$$
\begin{gathered}
\mathrm{M}=\max \left\{\left|\frac{\partial^{2} \hat{y}(k+1)}{\partial u(k)^{2}}\right|: u(k) \in \Omega\right\} \\
\mid \text { H.O.T } \mid \leq 0.5 \mathrm{M} u(k)^{2} \quad u(k) \in \Omega
\end{gathered}
$$

where $\Omega$ is the control domain embracing $u(k)=0$. By truncating the H.O.T. in (6), an affine NARMA model which represents a predictor is obtained as follows:

$$
\hat{y}_{\mathrm{a}}(k+1)=g_{0}(k)+g_{1}(k) \cdot u(k)
$$

where $\hat{y}_{\mathrm{a}}(k+1)$ is the prediction at time step $k+1$ given by the affine NARMA model. Obviously, (13) is not as accurate as (1) in predicting the plant output. However, the error between $\hat{y}_{\mathrm{a}}(k+1)$ and $\hat{y}(k+1)$ will be also limited if $|u(k)|$ is limited, according to (11) and (12).

Moreover, this error will become smaller as the decrease of $|u(k)|$. For example, when the system trends to the stable equilibrium at the late stage of control, $|u(k)|$ is quite small so that $\hat{y}_{\mathrm{a}}(k+1)$ is very close to $\hat{y}(k+1)$. Since (13) just neglects the high order terms with respect to $u(k)$, it will be more accurate than the linear ARMA model used in [13] for the control design which does not contain any high order terms.

In practice, when considering physical limits or trade-off of overall system dynamics, the maximum amplitudes of control inputs for various industrial plants are usually constrained to appropriate values. This means, in such case, the difference between (13) and (1) will be well limited and the predictive error of (13) will be quite acceptable, at least for the control purpose. Therefore, (13) is naturally employed to facilitate the control design since it is in a more tractable form (affine form). The proposed adaptive control scheme based on this model is introduced and applied to the design of APSS, which will be addressed in the next section.

\section{PROPOSED ADAPTIVE CONTROL SCHEME APPLIED TO DESIGN OF APSS}

To improve the objective system dynamic, it is expected that the output of the plant at time step $k+1$ is minimized by the control input at time step $k$. In this paper, if the amplitude of the plant control input is properly limited, $\hat{y}_{\mathrm{a}}(k+1)$ is assumed to be the plant output at time step $k+1$. Therefore the control input at time step $k$ can be obtained by solving the following minimization problem:

$$
\begin{gathered}
\min _{u(k)} J(k)=0.5 \hat{y}_{\mathrm{a}}(k+1)^{2}+0.5 \lambda u(k)^{2} \\
\text { s.t. } u_{\min } \leq u(k) \leq u_{\max } \\
\hat{y}_{\mathrm{a}}(k+1)=g_{0}(k)+g_{1}(k) \cdot u(k)
\end{gathered}
$$

where $u_{\min }$ and $u_{\max }$ are lower and upper limits, respectively, of the control input; $\lambda$ is the input weight factor with positive value used to optimize the overshoot and settling time of the response curve [17].

The solution of (14)-(16) can be analytically obtained as follows:

$$
u^{*}(k)=\left\{\begin{array}{lr}
u_{\max } & u_{\mathrm{c}}(k)>u_{\max } \\
u_{\mathrm{c}}(k) & u_{\min } \leq u_{\mathrm{c}}(k) \leq u_{\max } \\
u_{\min } & u_{\mathrm{c}}(k)<u_{\min }
\end{array}\right.
$$

with the following definition:

$$
u_{\mathrm{c}}(k)=-g_{1}(k) g_{0}(k) /\left(g_{1}(k)^{2}+\lambda\right)
$$

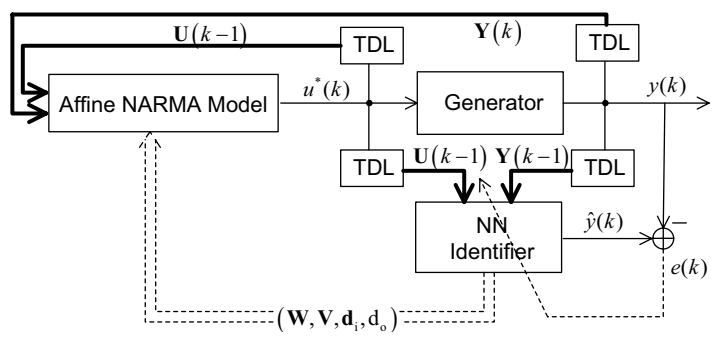

Fig. 2. The structure of AAPSS

One characteristic of $\mathrm{NN}$ identifier is its adaption capability. Depending on this, an adaptive control scheme based on the affine NARMA model is developed in this paper and applied to the design of APSS since the output of PSS is always confined within a quite small range [7]. The structure of AAPSS is shown in Fig. 2. Here the plant output is the speed deviation of the generator, while the control input is the supplementary exciting control input. TDL is the time-delay-line. The adaptive control scheme is divided into two stages, which are introduced in the following subsections.

\subsection{Off-line Training of NN Identifier}

A NN (Fig. 1) is trained off-line as identifier in this stage to capture the dynamic of the generator. Besides choosing a proper number of neurons in the hidden layer, a large amount of training data covering various operating conditions and disturbances is gathered to assure the generalization capability of the network [15]. The training data is collected by imposing pseudorandom sequences as probing signals to the generators to excite all modes of the system [8]. 


\subsection{Online Adaption and Control}

In this stage, the online learning capability of $\mathrm{NN}$ is utilized to cope with the unexpected variations of the system $[2,6,14,17]$. As illustrated in Fig. 2, the procedure of online adaption and control is presented as follows:

1) At time step $k$, sample $y(k)$;

2) If the error between $\hat{y}(k)$ and $y(k)$ is larger than the setting value, it is utilized to tune the weights and biases of the identifier by using error backpropagation algorithm [10];

3) The weights and biases are passed to the affine NARMA model. With samplings $\mathbf{U}(k-1)$ and $\mathbf{Y}(k), u^{*}(k)$ is computed by using (7)-(10), (17) and (18);

4) Apply $u^{*}(k)$ as the control input to the generator;

5) With samplings $\mathbf{U}(k)$ and $\mathbf{Y}(k)$, calculate $\hat{y}(k+1)$ by using $(1)$ and store it;

6) Let $k=k+1$, go to 1 ).

\section{COMPARISON OF AAPSS AND TAPSS}

If the predictor used in (16) is errorless, the solution of (14)-(16) will be the open loop optimal control input at time step $k$. However, if the NN based general NARMA model is used as predictor in (16), the solution (denoted by $u_{\mathrm{o}}^{*}(k)$ ) of (14)(16) will be very close to the open loop optimal control input because the predictive error of the $\mathrm{NN}$ identifier is quite small. Unfortunately, this solution cannot be online obtained analytically because of the special nonlinear relationship between $\hat{y}(k+1)$ and $u(k)$.

In TAPSS, the NN based general NARMA model is used as the predictor. Therefore, after training the identifier, a neuro-controller [2] or neuro-fuzzy controller [14] is generally employed in TAPSS to learn the optimal feedback control law off-line. It is expected that the control input generated by the resulted feedback control law at time step $k$ can approach $u_{\mathrm{o}}^{*}(k)$ as close as possible.

To learn the optimal feedback control law, the tunable parameters, i.e., weights of the controller are commonly tuned by applying the gradient descent method [6]. Obviously, this gradient based learning process may converge to a local minimum due to the infeasible initial values of the parameters. Moreover, the selection of tunable parameters considerably influences the resulted feedback control law [14]. Therefore, for these two considerations, the output of the controller at time step $k$ may be far from $u_{\mathrm{o}}^{*}(k)$. In AAPSS, as the amplitude of the PSS output is well limited, the difference between the affine NARMA model and the NN based general NARMA model is quite small. Therefore, the control input calculated based on the affine NARMA model is very close to $u_{\mathrm{o}}^{*}(k)$. Hence, it can be inferred that the AAPSS can always produce the optimal or the near optimal control input, while the TAPSS may not guarantee an optimal control performance. This means, theoretically speaking, the AAPSS generally performs better than the TAPSS.

The controller learning process of TAPSS should consider various operating conditions and disturbances to ensure its online control performance. Moreover, the backpropagation through time algorithm which converges slowly is generally employed for the learning process [9]. Therefore, the controller learning process of TAPSS is quite troublesome and time consuming. Fortunately, the proposed AAPSS does not require this step, which means the design process of the AAPSS is much simpler than that of the TAPSS.

\section{SIMULATION STUDIES}

\subsection{Single Machine Infinite Bus (SMIB) System}

The diagram of a SMIB system is shown in Fig. 3. The detailed models and parameters of this system are given in Appendix. The operating condition of the generator can be tuned by adjusting the voltage reference or the power reference or the shunt capacitor. All variables in this paper are expressed in per unit, if not specified.

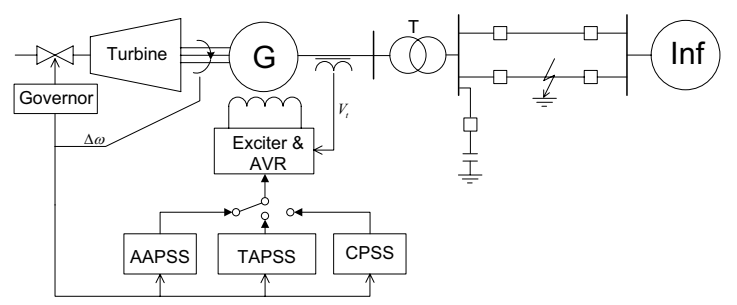

Fig. 3. SMIB system

An AAPSS is designed and equipped to the generator to damp the electromechanical oscillation. $\mathrm{m}, \mathrm{n}$ and $\mathrm{p}$ are set to 4, 4 and 10, respectively, for the NN identifier. This configuration can lead to a satisfactory control performance without increasing the complexity of the controller. The sampling period is chosen to be $30 \mathrm{~ms}$.

Firstly, the accuracy of the affine NARMA model in the AAPSS is validated. For this purpose, the following two indexes are defined:

$$
\begin{array}{cl}
\text { idx_1: } & \sum_{k=1}^{\mathrm{N}}\left|\left(\left|\hat{y}_{\mathrm{a}}(k)\right|+\varepsilon\right) /(|\hat{y}(k)|+\varepsilon)-1\right| / \mathrm{N} \\
\text { idx_2: } & \sum_{k=1}^{\mathrm{N}}\left|\hat{y}_{\mathrm{a}}(k)-y(k)\right| / \sum_{k=1}^{\mathrm{N}}|y(k)|
\end{array}
$$

where $\mathrm{N}$ is the total number of samplings; $\varepsilon$ is a quite small positive to avoid singularity when calculating idx 1 and here it is set to $1 \mathrm{e}-6$. Obviously, idx_1 represents the degree of 
similarity between the affine NARMA model and the NN based general NARMA model and as it is closer to zero, the difference between them gets smaller; idx_ 2 is the average relative predictive error of the affine NARMA model.

The generator is working in an initial condition of $P=0.6988, Q=0.1639$, and $V_{\mathrm{t}}=1.0523$, where $P, Q$ and $V_{\mathrm{t}}$ are active power output, reactive power output and terminal voltage, respectively, of the generator and it is subject to subsequent disturbances of a 0.2 step increase in the torque at $t=10 \mathrm{~s}$ and a 0.05 step decrease in the voltage reference at $t=20 \mathrm{~s}$. During this process, a pseudorandom sequence lasting for $30 \mathrm{~s}$ is applied as the supplementary exciting control input. Three different input sequences with maximum amplitudes $\left(\mathrm{A}_{\mathrm{m}}\right)$ of $0.05,0.10$ and 0.15 are generated for tests, respectively. For the three tests, idx 1 and idx 2 are calculated and given in Table I. It is seen that all $\mathrm{idx} \mathrm{x}_{-} 1$ are quite small and as $\mathrm{A}_{\mathrm{m}}$ become smaller, idx_1 is closer to zeros. This means the small difference between the affine NARMA model and the NN based general NARMA model, and it will decrease as the amplitude of control input becomes smaller. Furthermore, the small values of idx_ 2 imply the high accuracy of the affine NARMA model in predicting the output of the plant. This can be observed in Fig. 4 which illustrates two almost overlapped curves: one is the predicted curve of the generator speed deviation by the affine NARMA model and the other is the corresponding real curve, when using the pseudorandom sequence with $\mathrm{A}_{\mathrm{m}}=0.10$ as the control input. In this paper, the lower and upper limits for the supplementary exciting control are \pm 0.1 , respectively. Therefore, according to the analysis present in Section IV, the AAPSS will produce the control input that is very close to the real open loop optimal control input.

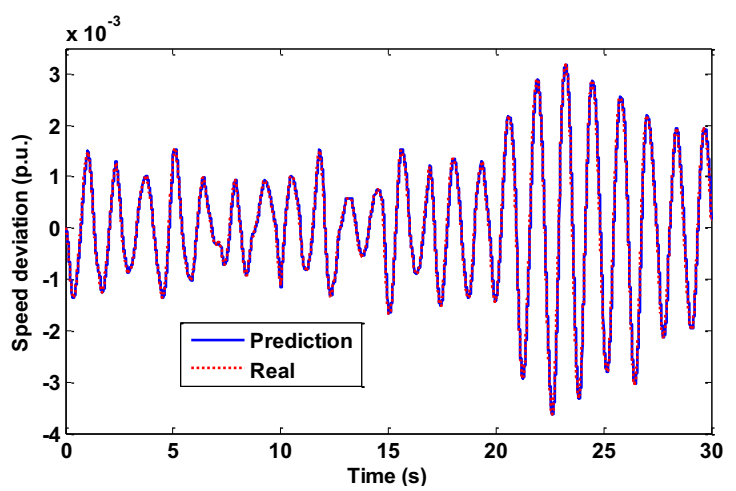

Fig. 4. Validation of proposed affine NARMA model
Table 1. idx_1 and idx_ 2 for different input sequences

\begin{tabular}{|r|r|r|r|}
\hline & $\mathrm{Am}=0.05$ & $\mathrm{Am}=0.10$ & $\mathrm{Am}=0.15$ \\
\hline idx_1 & $1.1746 \mathrm{e}-4$ & $2.9664 \mathrm{e}-4$ & $6.2216 \mathrm{e}-4$ \\
\hline idx_2 & $6.8223 \mathrm{e}-4$ & $4.9596 \mathrm{e}-4$ & $3.8620 \mathrm{e}-4$ \\
\hline
\end{tabular}

The online control performance of the AAPSS is now verified. As comparisons, a CPSS and a TAPSS are also employed. The CPSS is designed in the nominal operating condition of $P=0.8485$, $Q=0.2198$, and $V_{\mathrm{t}}=1.0510$ by using the phase compensation technique. The model and parameters of the CPSS are given in Appendix. A three-layer feedforward network with 10 neurons in its hidden layer is employed as the neuro-controller in the TAPSS. The control inputs at time step $k-1, k-2$ and $k-3$, together with the plant outputs at time step $k, k-1, k-2$ and $k-3$ are used as inputs to the neuro-controller to produce the control input at time step $k$.

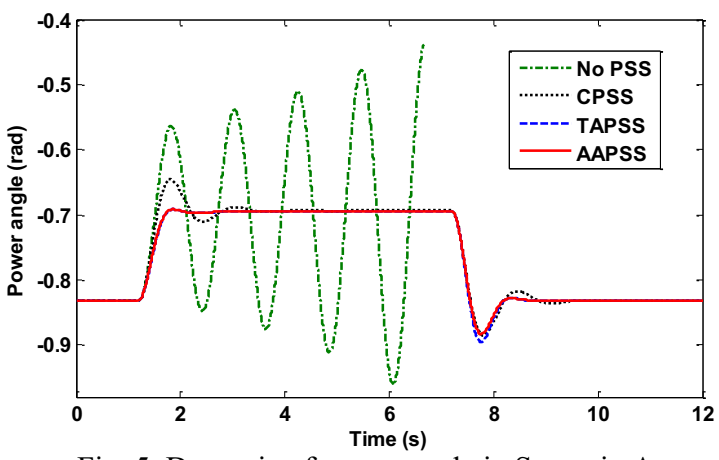

Fig. 5. Dynamic of power angle in Scenario A

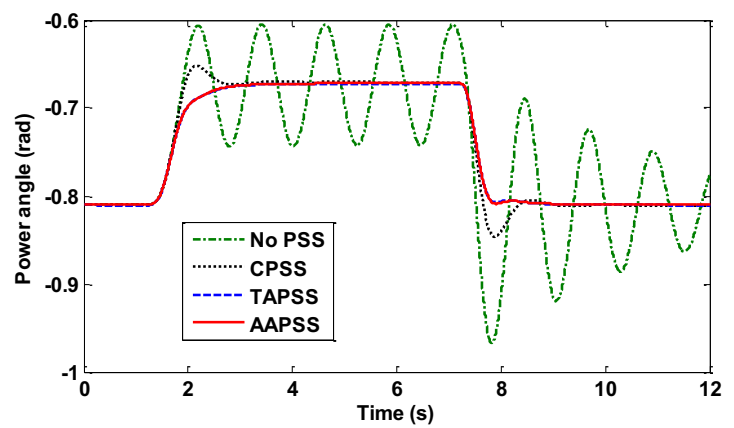

Fig. 6. Dynamic of power angle in Scenario B

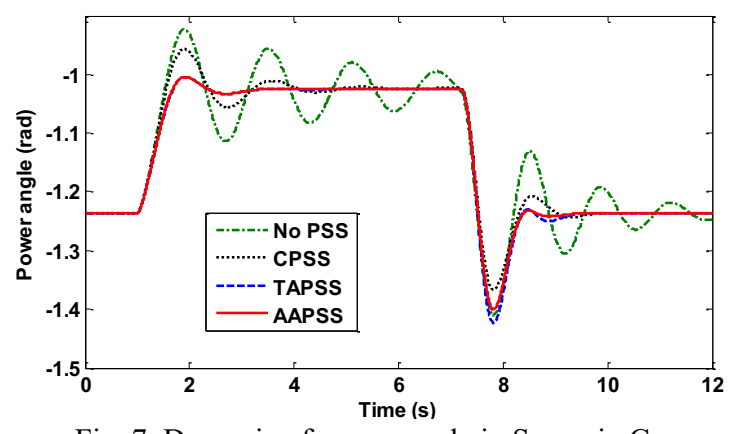

Fig. 7. Dynamic of power angle in Scenario C

The following scenarios are used for demonstrating the control performances of various PSSs: 
Scenario A: the generator is in the operating condition of $P=0.8485, Q=0.2198$, and $V_{t}=^{\prime} .0510$. A 0.15 step increase in the torque takes place at $t=1.2 \mathrm{~s}$ and it returns to initial value at $t=7.2 \mathrm{~s}$;

Scenario B: the generator is operating in the leading power factor condition of $P=0.6489$, $Q=-0.1753$, and $V_{\mathrm{t}}=1.0544$. A 0.05 step decrease in the voltage reference occurs at $t=1.2 \mathrm{~s}$ and it returns to initial value at $t=7.2 \mathrm{~s}$;

Scenario $C$ : the generator is working in the condition of $P=0.4994, Q=0.1123$, and $V_{\mathrm{t}}=1.0536$. A three phase short circuit fault takes place at the middle of one transmission line at $t=1.2 \mathrm{~s}$ and it is cleared by tripping the faulty line $50 \mathrm{~ms}$ later. The faulty line is then reclosed at $t=7.2 \mathrm{~s}$.

The power angle dynamics of the generator in the three scenarios are depicted in Fig. 5, Fig. 6 and Fig. 7, respectively. It is observed that the AAPSS is able to damp the oscillation of the generator rapidly in all scenarios and it generally outperforms the CPSS because the design of the CPSS does not consider the nonlinear and non-stationary characteristics of the system. Moreover, although the AAPSS has almost the same control performance as the TAPSS, the former actually performs slightly better than the latter (i.e., in Scenario A and Scenario C). Even so, the developing process of the AAPSS is much simpler than that of the TAPSS.

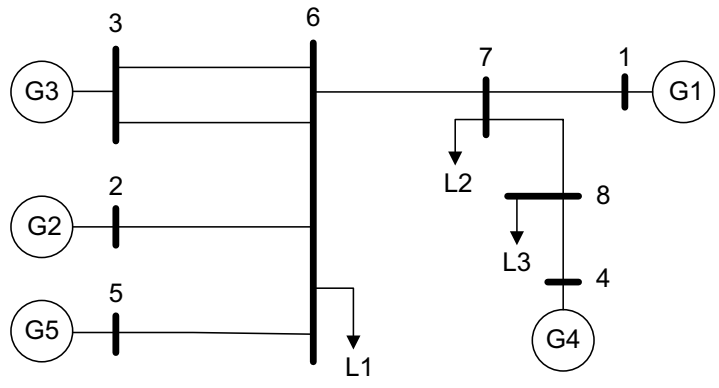

Fig. 8. Five-machine power system

\subsection{Multi-machine System}

A five-machine power system where there are multimode oscillations when it experiences disturbances is illustrated in Fig. 8 [18]. An interarea oscillation with frequency at about 0.64 $\mathrm{Hz}$ exists between the area with Generators G2, G3 and G5 and the area with Generators G1 and G4. Moreover, Oscillations of local mode with higher frequencies reside in each area. The models and parameters in detail of this system are given in the Appendix.

To damp the oscillations, the AAPSS can be designed for each generator when the PSS control loops of other generators are open. The coordination of these AAPSSs is fulfilled by their online adaption capabilities. $\mathrm{m}, \mathrm{n}$ and $\mathrm{p}$ are set to 4,4 and 10 , respectively, for the $\mathrm{NN}$ identifiers in all the AAPSSs. The sampling period is selected to be $40 \mathrm{~ms}$. Two operating conditions which are involved in the Appendix are employed for simulation studies. The upper and lower limits for the PSS output are set to \pm 0.1 , respectively, for all the generators.
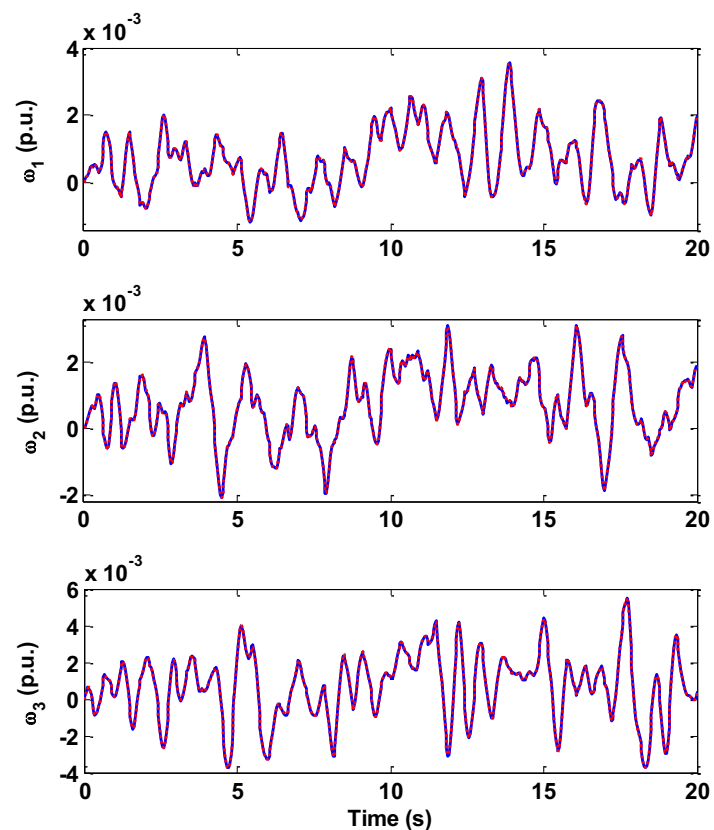

Fig. 9. Predictions of affine NARMA models (solid line: predictive; dot line: real)

The accuracy of the affine NARMA models used in the AAPSSs is verified. As the system is in operating condition OP1, apply pseudorandom sequences as the supplementary exciting control inputs to the five generators, simultaneously. The predictions of the affine NARMA models for Generators G1, G2 and G3 are shown in Fig.9. Obviously, the almost overlapped curves indicate a pretty accuracy of the affine NARMA models in predicting the outputs of these generators. In fact, the affine NARMA models can also accurately track the outputs of Generator G4 and G5, but for space limitation they are not displayed. Therefore, the highly accurate affine NARMA models can theoretically ensure that the AAPSSs generally perform better than the TAPSSs.

CPSSs are then employed as comparisons to the AAPSSs and each CPSS is designed in operating condition OP1 by using the phase compensation technique when other PSSs are out of service. The parameters of the CPSSs are included in the Appendix. The following testing scenarios are utilized to validate the online control performance of the AAPSSs:

Scenario A: PSSs (AAPSSs or CPSSs) are only installed in Generator G1, G2 and G3 as the system is operating in OP1. A 0.3 step decrease in the 
torque of Generator G3 occurs at $t=2 \mathrm{~s}$ and it returns to initial value at $t=11 \mathrm{~s}$;
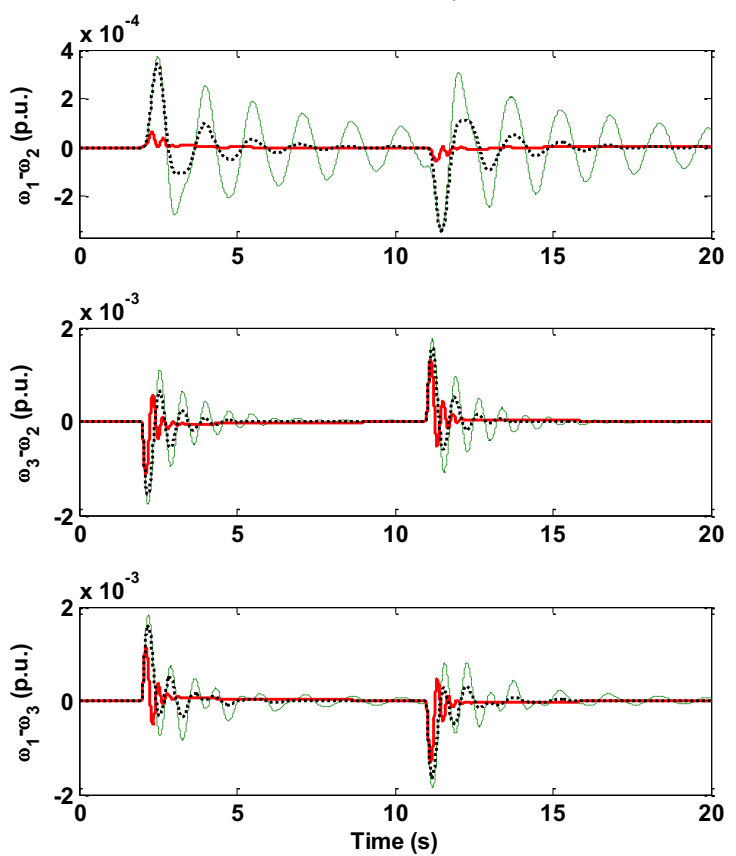

Fig. 10. System responses in Scenario A (solid line: AAPSSs; dot line: CPSSs; dash line: no PSS)
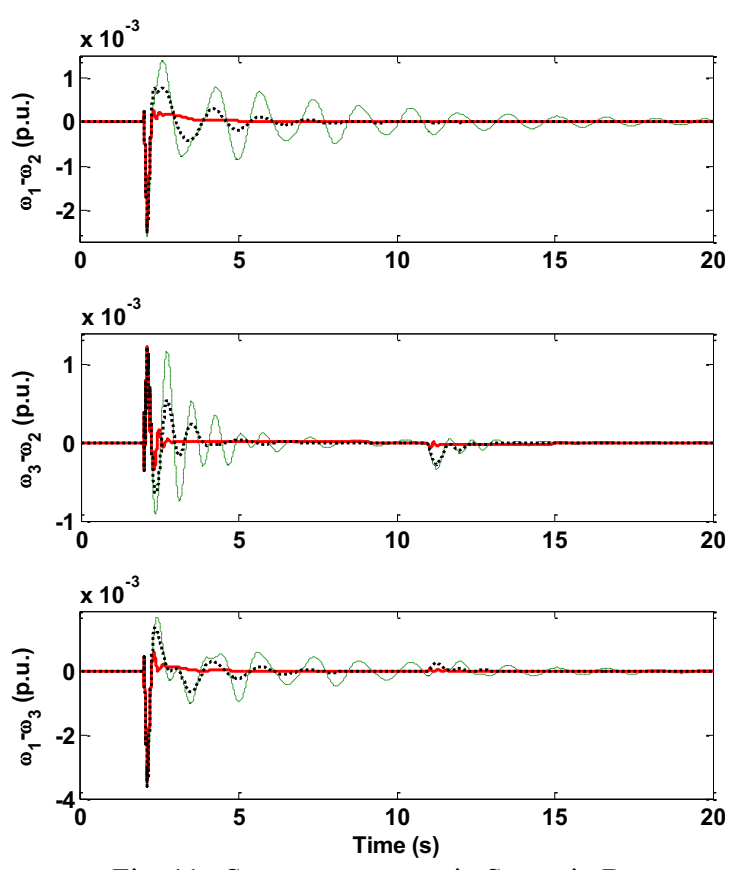

Fig. 11. System responses in Scenario B (solid line: AAPSSs; dot line: CPSSs; dash line: no PSS)
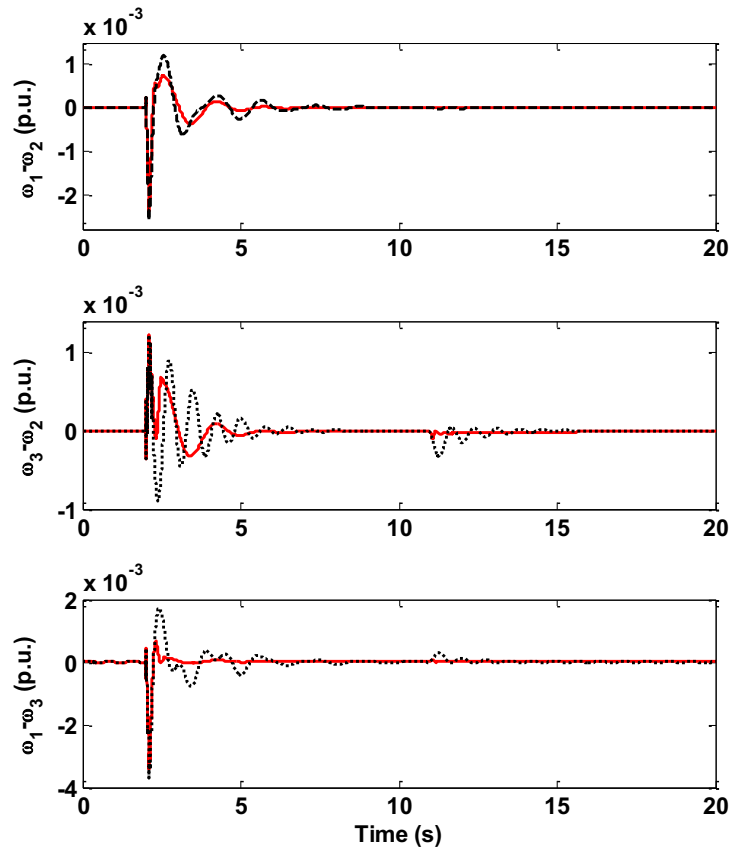

Fig. 12. System responses in Scenario C (solid line: AAPSSs and CPSSs; dot line: CPSSs)

Scenario B: this scenario is the same as Scenario A except for different disturbances. A three phase short circuit fault occurs at the middle of one transmission line between Bus 3 and 6 at $t=2 \mathrm{~s}$ and is cleared by tripping the faulty line $100 \mathrm{~ms}$ later. The line is then re-energied at $t=11 \mathrm{~s}$;

Scenario C: the system is in the same operating condition and subject to the same disturbances as those in Scenario B. However here, firstly only CPSSs are equipped to Generators G2, G4 and G5. Then, as a comparative case, the AAPSSs are additionally installed in Generators G1 and G3 to cooperate with the existing CPSSs;

Scenario D: the system is in operating condition OP2, while PSSs (AAPSSs or CPSSs) are only installed in Generator G1, G2 and G3. A 0.3 step decrease in the torque of Generator G3 occurs at $t=2 \mathrm{~s}$ and it returns to initial value at $t=11 \mathrm{~s}$.

The system responses in these scenarios are illustrated in Fig. 10, Fig. 11, Fig. 12 and Fig. 13, respectively. It is clear that the AAPSSs can cooperatively perform to rapidly damp the oscillations in the system in despite of the variation of operating condition and the different disturbances (Scenario A, Scenario B and Scenario D). The performance of the AAPSSs is apparently better than that of the CPSSs because each CPSS is merely linearly designed in an independent manner around a specific operating point. Moreover, because of their online learning capabilities, the AAPSSs are also capable of coordinating with the existing CPSSs to further improve the dynamics of the system (Scenario C). 

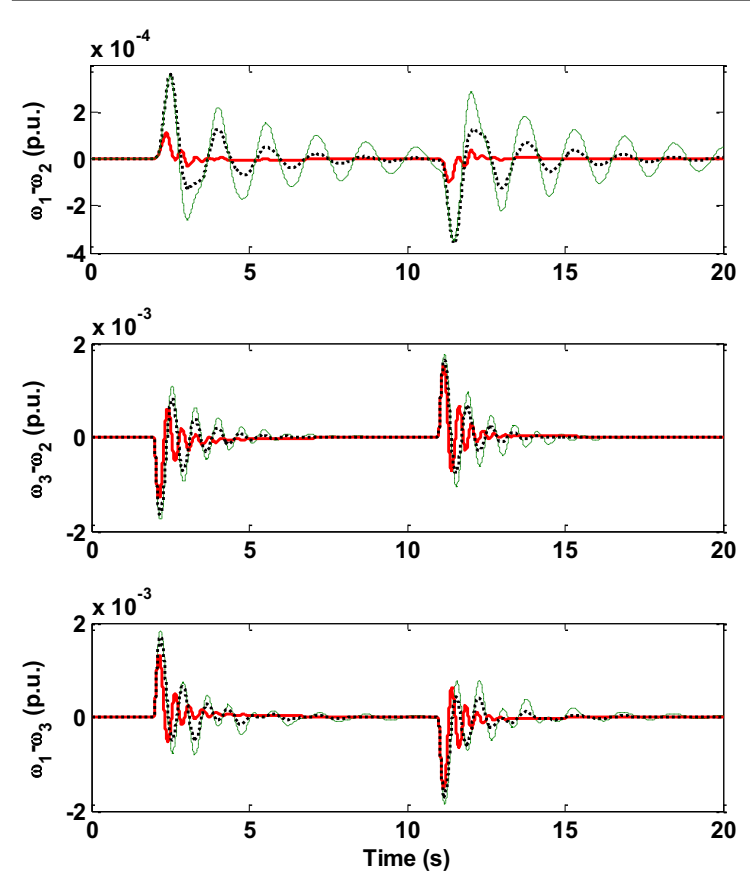

Fig. 13. System responses in Scenario D (solid line: AAPSSs; dot line: CPSSs; dash line: no PSS)

\section{CONCLUSION}

An affine NARMA model obtained by performing Taylor series expansion on the $\mathrm{NN}$ based general NARMA model has been proposed for the control design. This approximate model is accurate enough to predict the plant output for the purpose of control design if the amplitude of the control input is appropriately limited. Consequently, based on this model, an adaptive control scheme has been proposed to online analytically calculate the control input. This control scheme has been suitably applied to the design of APSS because the amplitude of PSS output is always well limited. Theoretical analysis has shown that the AAPSS generally outperforms the TAPSS and the developing process of the AAPSS is also much simpler than that of the TAPSS. Simulation results on a SMIB system and a multi-machine system have verified the high accuracy of the affine NARMA models used for the AAPSSs. It has also been demonstrated that the AAPSSs can well perform to adaptively damp the electromechanical oscillations in the systems and their performances are generally better than those of the CPSSs.

\section{APPENDIX}

\subsection{Models}

1) The synchronous machine with round rotor in the SMIB system is represented by a six-order model. All synchronous machines in the multimachine system are salient-pole machines and represented by five-order models [7].

2) The excitation systems in both systems consist of an IEEE type 1 synchronous machine voltage regulator combined to an exciter [20].

3) The turbines and governors are presented by the following simplified model [2]:

$$
g=\left(a+b /\left(1+s T_{g}\right)\right) \omega
$$

4) The CPSS has the following transfer function:

$$
V_{\mathrm{pss}}=K_{\mathrm{p}}\left(\frac{s T_{\mathrm{w}}}{1+s T_{\mathrm{w}}}\right)\left(\frac{1+s T_{1}}{1+s T_{2}}\right)\left(\frac{1+s T_{3}}{1+s T_{4}}\right) \omega
$$

\subsection{Parameters}

The fundamental frequency for both systems is $60 \mathrm{~Hz}$. The benchmark power is 900-MVA for the SMIB system and 100-MVA for the multi-machine system. The detailed parameters are listed in the following tables.

Table 2.

Parameters of generators in multi-machine system

\begin{tabular}{|c|c|c|c|c|c|}
\hline & $\mathrm{G} 1$ & $\mathrm{G} 2$ & $\mathrm{G} 3$ & $\mathrm{G} 4$ & $\mathrm{G} 5$ \\
\hline$x_{\mathrm{d}}$ & 0.1026 & 0.1026 & 1.0260 & 0.1026 & 1.0260 \\
\hline$x_{\mathrm{q}}$ & 0.0658 & 0.0658 & 0.6580 & 0.0658 & 0.6580 \\
\hline$x_{\mathrm{d}}{ }^{\prime}$ & 0.0339 & 0.0339 & 0.3390 & 0.0339 & 0.3390 \\
\hline$x_{\mathrm{d}}{ }^{\prime \prime}$ & 0.0335 & 0.0335 & 0.3350 & 0.0335 & 0.3350 \\
\hline$x_{\mathrm{q}}{ }^{\prime}$ & 0.0335 & 0.0335 & 0.3350 & 0.0335 & 0.3350 \\
\hline$x_{1}$ & 0.0094 & 0.0094 & 0.0940 & 0.0094 & 0.0940 \\
\hline$T_{\mathrm{d} 0}{ }^{\prime}(\mathrm{s})$ & 5.6700 & 5.6700 & 5.6700 & 5.6700 & 5.6700 \\
\hline$T_{\mathrm{d} 0}{ }^{\prime}(\mathrm{s})$ & 0.6140 & 0.6140 & 0.6140 & 0.6140 & 0.6140 \\
\hline$T_{\mathrm{q} 0}{ }^{\prime}(\mathrm{s})$ & 0.7230 & 0.7230 & 0.7230 & 0.7230 & 0.7230 \\
\hline$H_{(\mathrm{s})}$ & 80.000 & 80.000 & 10.000 & 80.000 & 10.000 \\
\hline$T_{\mathrm{r}}(\mathrm{s})$ & 0.04 & 0.04 & 0.04 & 0.04 & 0.04 \\
\hline$K_{\mathrm{a}}$ & 190 & 190 & 190 & 190 & 190 \\
\hline$T_{\mathrm{a}}(\mathrm{s})$ & 0.001 & 0.001 & 0.001 & 0.001 & 0.001 \\
\hline$E_{\text {fmin }}$ & -11.5 & -11.5 & -11.5 & -11.5 & -11.5 \\
\hline$E_{f \max }$ & 11.5 & 11.5 & 11.5 & 11.5 & 11.5 \\
\hline$a$ & $-2 \mathrm{e}-4$ & 0.0002 & 0.0015 & 0.0002 & $-1.5 \mathrm{e}-4$ \\
\hline$b$ & 0.025 & 0.025 & -0.20 & 0.025 & -0.20 \\
\hline$T_{\mathrm{g}}(\mathrm{s})$ & 0.28 & 0.28 & 0.28 & 0.28 & 0.28 \\
\hline$T_{\mathrm{w}}(\mathrm{s})$ & 10 & 10 & 10 & 10 & 10 \\
\hline$T_{1}(\mathrm{~s})$ & 0.2957 & 0 & 0.2626 & 0.2017 & 0.2588 \\
\hline$T_{2}(\mathrm{~s})$ & 0.2082 & 0 & 0.0522 & 0.1276 & 0.0563 \\
\hline$T_{3}(\mathrm{~s})$ & 0 & 0 & 0.2626 & 0 & 0.2588 \\
\hline$T_{4}(\mathrm{~s})$ & 0 & 0 & 0.0522 & 0 & 0.0563 \\
\hline $\mathrm{K}_{\mathrm{p}}$ & 50 & 8 & 1.5 & 7 & 2 \\
\hline $\mathrm{V}_{\max }$ & 0.1 & 0.1 & 0.1 & 0.1 & 0.1 \\
\hline $\mathrm{V}_{\min }$ & -0.1 & -0.1 & -0.1 & -0.1 & -0.1 \\
\hline & & & & & \\
\hline
\end{tabular}

Table 3.

Parameters of generators in SMIB system

\begin{tabular}{|c|c|c|c|c|c|}
\hline$x_{\mathrm{d}}$ & 1.800 & $x_{\mathrm{q}}$ & 1.700 & $T_{\mathrm{d} 0}{ }^{\prime}(\mathrm{s})$ & 8.000 \\
\hline$x_{\mathrm{d}}{ }^{\prime}$ & 0.300 & $x_{\mathrm{q}}{ }^{\prime}$ & 0.550 & $T_{\mathrm{d} 0}{ }^{\prime}(\mathrm{s})$ & 0.030 \\
\hline$x_{\mathrm{d}}{ }^{\prime}$ & 0.250 & $x_{\mathrm{q}}{ }^{\prime}$ & 0.250 & $T_{\mathrm{q} 0}{ }^{\prime}(\mathrm{s})$ & 0.400 \\
\hline$x_{1}$ & 0.200 & $H(\mathrm{~s})$ & 8.500 & $T_{\mathrm{q} 0}{ }^{\prime \prime}(\mathrm{s})$ & 0.050 \\
\hline$K_{\mathrm{a}}$ & 200 & $T_{\mathrm{a}}(\mathrm{s})$ & 0.001 & $T_{\mathrm{r}}(\mathrm{s})$ & 0.02 \\
\hline$E_{\text {fmin }}$ & -10 & $T_{\mathrm{g}}(\mathrm{s})$ & 0.17 & $T_{\mathrm{w}}(\mathrm{s})$ & 10 \\
\hline$E_{\text {fmax }}$ & 10 & $T_{1}(\mathrm{~s})$ & 0.2778 & $T_{2}(\mathrm{~s})$ & 0.1414 \\
\hline $\mathrm{K}_{\mathrm{p}}$ & 16 & $T_{3}(\mathrm{~s})$ & 0 & $T_{4}(\mathrm{~s})$ & 0 \\
\hline $\mathrm{V}_{\max }$ & 0.1 & $a$ & 0.002 & - & - \\
\hline $\mathrm{V}_{\min }$ & -0.1 & $b$ & -0.20 & - & - \\
\hline
\end{tabular}


Table 4.

Parameters of Transmission Lines

\begin{tabular}{|c|c|c|c|c|}
\hline $\begin{array}{c}\text { From } \\
\text { Bus }\end{array}$ & $\begin{array}{c}\text { To } \\
\text { Bus }\end{array}$ & $r$ & $x$ & $B$ \\
\hline 1 & 7 & 0.00435 & 0.01067 & 0.03072 \\
\hline 2 & 6 & 0.00213 & 0.00468 & 0.00808 \\
\hline 3 & 6 & 0.01002 & 0.03122 & 0.06408 \\
\hline 3 & 6 & 0.01001 & 0.03122 & 0.06408 \\
\hline 4 & 8 & 0.00524 & 0.01184 & 0.03530 \\
\hline 5 & 6 & 0.00711 & 0.02331 & 0.05464 \\
\hline 6 & 7 & 0.04032 & 0.12785 & 0.31716 \\
\hline 7 & 8 & 0.01724 & 0.04153 & 0.12028 \\
\hline $1^{*}$ & $2^{*}$ & 0.07200 & 0.72000 & 0.24500 \\
\hline $1^{*}$ & $2^{*}$ & 0.07200 & 0.72000 & 0.24500 \\
\hline
\end{tabular}

Table 5 .

Operating Conditions for Multi-machine System

\begin{tabular}{|c|c|c|c|c|c|c|}
\hline \multicolumn{2}{|c|}{} & $\mathrm{G} 1$ & $\mathrm{G} 2$ & $\mathrm{G} 3$ & $\mathrm{G} 4$ & $\mathrm{G} 5$ \\
\hline \multirow{3}{*}{ OP1 } & $P$ & 5.133 & 8.583 & 0.8055 & 8.567 & 0.8501 \\
\cline { 2 - 8 } & $Q$ & 6.796 & 4.422 & 0.4050 & 4.662 & 0.2062 \\
\cline { 2 - 7 } & $V_{\mathrm{t}}$ & 1.075 & 1.05 & 1.025 & 1.075 & 1.025 \\
\hline \multirow{3}{*}{ OP2 } & $P$ & 3.168 & 4.103 & 0.4708 & 4.068 & 0.1647 \\
\cline { 2 - 7 } & $Q$ & 2.923 & 1.405 & 0.4091 & 2.189 & 0.3406 \\
\cline { 2 - 7 } & $V_{\mathrm{t}}$ & 1.050 & 1.030 & 1.025 & 1.050 & 1.025 \\
\hline
\end{tabular}

Table 6.

Load Admittance

\begin{tabular}{|c|c|c|c|}
\hline & L1 & L2 & L3 \\
\hline OP1 & $7.5-j 5.0$ & $8.5-j 5.0$ & $7.0-j 4.0$ \\
\hline OP2 & $3.755-j 2.5$ & $4.25-j 2.5$ & $3.5-j 2.25$ \\
\hline
\end{tabular}

\section{REFERENCES}

1. Chaturvedi DK, Malik OP, Kalra PK. Performance of a generalized neuron-based PSS in a multi-machine power system. IEEE Trans. Energy Convers, 2004; 19(3): 625-632.

http://dx.doi.org/10.1109/TEC.2004.827706.

2. Chaturvedi DK, Malik OP. Generalized neuron-based adaptive PSS for multi-machine environment. IEEE Trans Power Syst, 2005; 20(1): 358-366. http://dx.doi.org/10.1109/TPWRS.2004.840410.

3. Chaturvedi DK, Malik OP. Neurofuzzy power system stabilizer. IEEE Trans Energy Convers, 2008; 23(3): 887-894. http://dx.doi.org/10.1109/TEC.2008.918633.

4. Chung CY, Wang KW, Tse CT, Riu N. PSS design by probabilistic sensitivity indices. IEEE Trans Power Systs, 2002; 17(3): 688-693. http://dx.doi.org/10.1109/PESS.2002.1043544.

5. Deng H, Li HX, Wu YH. Feedback-linearizationbased neural adaptive control for unknown nonaffine nonlinear discrete-time systems. IEEE Trans Neural Netw, 2008 19(9): 1615-1625. http://dx.doi.org/10.1109/TNN.2008.2000804.

6. He J, Malik OP. An adaptive power system stabilizer based on recurrent neural networks. IEEE Trans Energy Convers, 1997; 12(4): 413-418. http://dx.doi.org/10.1109/60.638966.

7. Kundar P. Power System Stability and Control. New York: McGraw-Hill, 1994.

8. Mohagheghi S, Venayagamoorthy GK, Harley RG. Optimal wide area controller and state predictor for a power system. IEEE Trans Power Syst, 2007; 22(2): 693-705.

http://dx.doi.org/10.1109/TPWRS.2007.895158.
9. Narendra KS, Mukhopadhyay S. Adaptive control using neural networks and approximate models. IEEE Trans Neural Netw, 1997; 8(3): 475-485. http://dx.doi.org/10.1109/72.572089.

10. Nguyen TT, Gianto R. Neural networks for adaptive control coordination of PSSs and FACTS devices in multi-machine power system. IET Gener Transm Distrib, 2007; 2(3): 355-372. http://dx.doi.org/10.1049/iet-gtd:20070125.

11. Park JW, Venayagamoorthy GK, Harley RG. MLP/RBF neural-networks-based online global model identification of synchronous generator. IEEE Trans Ind Electron, 2005; 52(6): 1685-1695. http://dx.doi.org/10.1109/TIE.2005.858703.

12. Park YM, Moon UC, Lee KY. A self-organizing power system stabilizer using fuzzy auto-regressive moving average (FARMA) model. IEEE Trans Energy Convers, 1996; 11(2): 442-448. http://dx.doi.org/10.1109/60.507658.

13. Ramakrishna G, Malik OP. Radial basis function identifier and pole-shifting controller for power system stabilizer application. IEEE Trans Energy Convers, 2004; 19(4): 663-670. http://dx.doi.org/10.1109/TEC.2004.837268.

14. Ramirez-Gonzalez M, Malik OP. Power system stabilizer design using an online adaptive neurofuzzy controller with adaptive input link weights. IEEE Trans Energy Convers, 2008; 23(3): 914-922. http://dx.doi.org/ 10.1109/TEC.2008.921465.

15. Sarangapani J. Neural network control of nonlinear discrete-time systems. Boca Raton: CRC Press; 2006.

16. Segal R, Kothari ML, Madnani S. Radial basis function (RBF) network adaptive power system stabilizer. IEEE Trans Power Syst, 2000; 15(2): 722727. http://dx.doi.org/10.1109/PESW.2000.850186.

17. Shamsollahi P, Malik OP. An adaptive power system stabilizer using on-line trained neural networks. IEEE Trans Energy Convers, 1987; 12(4): 382-387. http://dx.doi.org/10.1109/60.638951.

18. Wu B, Malik OP. Multivariable adaptive control of synchronous machines in a multi-machine power system. IEEE Trans Power Syst, 2006; 21(4): 17721781. http://dx.doi.org/10.1109/TPWRS.2006.882454.

19. You R, Eghbali HJ, Nehrir MH. An online adaptive neuro-fuzzy power system stabilizer for multimachine systems. IEEE Trans Power Syst, 2003; 18(1): 128-135.

http://dx.doi.org/10.1109/TPWRS.2002.804961.

20. Zhang Y, Chen GP, Malik OP, Hope GS. An artificial neural network based adaptive power system stabilizer. IEEE Trans. Energy Convers, 1993; 8(1): 71-77. http://dx.doi.org/10.1109/60.207408.

\section{Received 2017-12-25}

Accepted 2018-01-23

Available online 2018-04-26

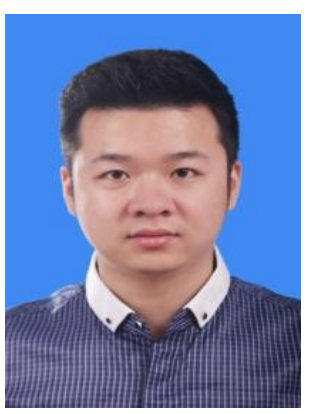

Jingsen ZHOU received the B.S degree in Electrical Engineering in 2012 from Wuhan University, Wuhan, China. Currently, he is pursuing the $\mathrm{Ph} . \mathrm{D}$. degree in the School of Electrical Engineering, Wuhan University. From 2016 to 2017, he is funded as a joint $\mathrm{Ph} . \mathrm{D}$. student in the Department of Electrical 
and Computer Engineering at the University of Saskatchewan, Saskatoon, SK, Canada. His research interests are in power system stability and control, wind power.

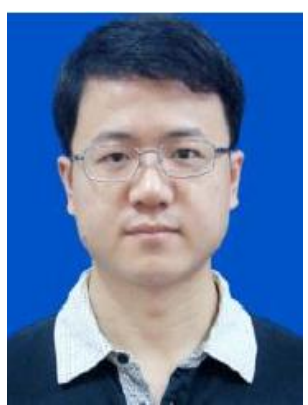

Deping KE received the B.S degree in Electrical Engineering in 2005 from Huazhong University of Science and Technology, Wuhan, China, and the Ph.D. degree in Electrical Engineering from The Hong Kong Polytechnic University in 2012. Currently, he is an Associate Professor with the School of Electrical Engineering, Wuhan University, China. His research interests are in power system dynamics and control, and economic operation of power systems.

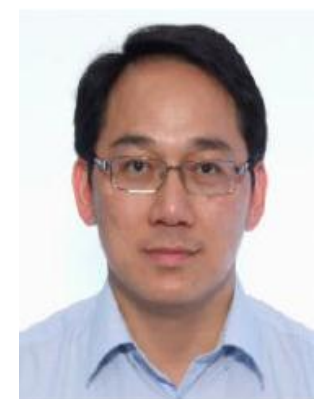

Chi Yong CHUNG (M'01SM'07-F'16) received the Ph.D. degree in electrical engineering from The Hong Kong Polytechnic University in 1999. He is currently a Professor and the SaskPower Chair in Power Systems Engineering in the Department of Electrical and Computer Engineering at the University of Saskatchewan, Saskatoon, SK, Canada. His research interests include power system stability/control, planning and operation, computational intelligence applications, power markets, and electric vehicle charging. Dr. Chung is an Editor of the IEEE Transactions on Sustainable Energy and the IEEE Transactions on power systems. He is also an Editorial Board Member of IET Generation, Transmission \& Distribution. He is also a Member-atLarge (Smart Grid) of IEEE PES Governing Board and the IEEE PES Region 10 North Chapter Representative.

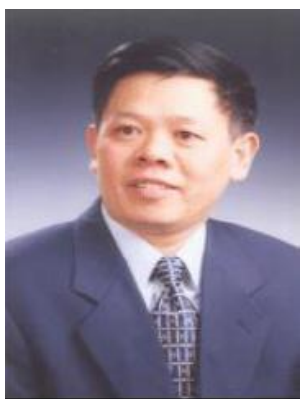

Yuanzhang SUN (M'99SM'01) received the B.S. degree from Wuhan University of Hydro and Electrical Engineering, Wuhan, China, in 1982, the M.S. degree from the Electric Power Research Institute(EPRI), Beijing, China, in 1984, and the Ph.D. degree in electrical engineering from Tsinghua University, Beijing, in 1988. Currently, he is a Professor of the School of Electrical Engineering at Wuhan University, and a Chair Professor of the Department of Electrical Engineering and Vice Director of the State Key Lab of Power System Control and Simulation at Tsinghua University. His main research interests are in the areas of power system dynamics and control, wind power, voltage stability and control, and reliability. 